\title{
BMJ Open Semiqualitative research protocol to explore cancer care workforce perceptions of the health system response to COVID-19 preparations in Southeast Queensland, Australia
}

\author{
Harry Gasper (D) ,1,2,3 Elizabeth Ahern, ${ }^{1,2,4,5}$ Natasha Roberts, ${ }^{1,6}$ Bryan Chan, ${ }^{2,7}$ \\ Brett Hughes, ${ }^{1,2}$ Glen Kennedy, ${ }^{1,2}$ David Wyld, ${ }^{1,2}$ Melissa Eastgate, ${ }^{1,2}$ Zarnie Lwin ${ }^{1,2}$
}

To cite: Gasper $\mathrm{H}$, Ahern E, Roberts N, et al. Semiqualitative research protocol to explore cancer care workforce perceptions of the health system response to COVID-19 preparations in Southeast Queensland, Australia. BMJ Open 2021;11:e044655. doi:10.1136/ bmjopen-2020-044655

- Prepublication history and additional online supplemental material for this paper are available online. To view these files, please visit the journal online (http://dx.doi.org/10. 1136/bmjopen-2020-044655).

Received 09 September 2020 Revised 16 December 2020 Accepted 23 March 2021
Check for updates

(C) Author(s) (or their employer(s)) 2021. Re-use permitted under CC BY-NC. No commercial re-use. See rights and permissions. Published by BMJ.

For numbered affiliations see end of article.

Correspondence to

Dr Harry Gasper;

harry.gasper@health.qld.gov.au

\section{ABSTRACT}

Introduction Sars-CoV-2 is a novel coronavirus responsible for COVID-19 officially declared pandemic in March 2020. Health systems worldwide responded with swift changes to increase workflow capacity while protecting the vulnerable, including those with cancer. This led to unprecedented and rapid restructuring of health service provision. Published data from the 2003 SARS pandemic focuses on medical and nursing staff, overlooking other departmental employees such as administration officers or food service workers. Our protocol aims to document directives and adjustments communicated to staff in two cancer care departments and correlate this with measures of distress and perceived preparedness across the spectrum of all staff involved in cancer care.

Methods and analysis We use a semiqualitative approach comprising weekly diarising of events and simultaneous staff surveys. Principal investigators will document changes at a metropolitan quaternary cancer centre and a regional cancer centre. Communications, directives and changes will be diarised in real time in four executional domains. Simultaneously, prospective voluntary selfadministered online surveys will be conducted at regular intervals by staff. The survey assesses the perceived institutional preparedness and personal well-being, with a combination of Likert scaled and open response questions. A semiquantitative self-assessment of distress adapted from National Comprehensive Cancer Network distress thermometer is incorporated. Additionally, opentext personal reflections on themes including difficult decisions will be invited. Survey participants will be drawn from various work areas of the cancer care departments: administrative staff, health professionals, for example, allied health, ancillary workers, nursing and medical.

Ethics and dissemination The study has been reviewed and approved by the Human Research Ethics Committee (LNR/2020/QRBW/62982). Published literature on domains of distress neglects categories of healthcare worker who form an essential part of the care delivery team. Our study hopes to gather insights about psychosocial impact and adjustment which could direct responses in future emergencies.
Strengths and limitations of this study

- This is the only study to our knowledge that explores impact on not only doctors and nurses, but also allied health, administrative and ancillary staff with patients facing roles.

- Our study highlights cancer care services as an allinclusive team, where we are all in this together.

- The swift and timely approval and implementation of our study allows us to collect prospective data from the outset through escalation, peak, recovery and subsequent waves.

- The main limitation can be survey fatigue and attrition due to the uncertain time frame of the pandemic.

\section{INTRODUCTION}

Health system stressors from COVID-19

Health services worldwide have rapidly responded to the spread of a novel coronavirus, SARS-CoV-2, which gives rise to the illness COVID-19, and was officially declared a pandemic by WHO on 11 March $2020 .^{12}$ Infection with the virus is associated with a mild illness for most patients; however, around 20\% develop a more severe syndrome, with risks of progression to acute respiratory distress syndrome, admission to intensive care, and death in up to $1 \%-10 \%$ of infected patients. ${ }^{3}$ Early reports suggested that older age and the presence of comorbidity, including cancer, were risk factors predictive of severe disease and death among those patients admitted to hospital. ${ }^{3-9}$ In addition to direct health risks from viral exposure, indirect hazards arising from COVID-19 have been identified that may result in further adverse oncologic outcomes. These include effects of social distancing and substituting face-to-face outpatient contact with telehealth. Although previous literature supports 
positive responses to telehealth, ${ }^{10}$ patient and healthcare worker (HCW) satisfaction with rapid transfer to telehealth as part of remote service provision in the context of a pandemic is unknown. Furthermore, in the event of personnel shortages or system overrun, rationalisation of service provision is projected, ${ }^{11}$ which would lead to real-time capacity testing of supportive care services such as palliative care and primary care physicians. ${ }^{12}$ Other collateral system impacts include constraints on the provision of clinical trials which would limit cancer patients' access to emerging therapeutics. ${ }^{13}$ Adverse oncological outcomes may also result from delayed investigation of symptoms arising from a cancer presentation, or requirements to temporise definitive management. ${ }^{14}$ Taken together, health system stressors combined with several direct and indirect risks arising from COVID-19 threaten the continuity and quality of cancer care.

Additionally, specific risks exist for the wider cancer healthcare workforce in the operational response to COVID-19. ${ }^{15}$ As COVID-19 case numbers increase, intense media speculation may distract from management of patients with cancer or symptoms suggestive of cancer. ${ }^{16}$ COVID-19 is likely to have wide-reaching consequences on the delivery of cancer care which might extend beyond the period of the peak infection, and long into the recovery period.

\section{Psychosocial stressors}

Alongside the operational stressors, several psychosocial consequences to HCW can be predicted based on the published experience from previous pandemics such as the previous SARS outbreak arising from a previous epidemic coronavirus in 2003. ${ }^{17-19}$ Pressures on staff were reported in Singapore and Toronto during this SARS epidemic and included concerns about risk of infection, possible stigmatisation, risks to family, isolation and guilt. ${ }^{1720}$ As resource scarcity increases during the current pandemic, there are predictions of moral hazards to HCW akin to those encountered in armed conflict. ${ }^{21}$ Cancer care staff face a difficult task balancing the protection of patients from a potentially deadly infection, providing equitable and safe care and managing their own personal distress.

\section{System preparedness}

An increasingly voluminous literature has been produced in a short time describing various approaches to preparing for a pandemic. This is generally limited to expert opinion or institutional guidelines ${ }^{21-24}$ but has fostered a collaborative environment to share ideas and strategies. Additionally, although a global event, the previous luxury of international travel and easy communication has been lost, with the concurrent cancellation of international conferences and events. Attention has therefore been drawn also to the need for collaboration between regional partners. ${ }^{25}$ Literature related to previous outbreaks of Middle Eastern respiratory syndrome and SARS can be drawn on, which need to be assessed and applied within the framework of the healthcare system in which the responses occurred. ${ }^{22} 23$ 26-28 But there will be a pressing need for experiences to be recorded and shared, as the situation evolves.

While literature exists regarding the impact of previous pandemic events on front-line clinical personnel such as doctors and nurses, a paucity of information relates to the risks of distress among other members of the HCW team such as allied health professionals, administrative staff and ancillary staff. The common finding from the 2003 SARS experience was that those who have most direct contact with patients (eg, nurses), have the highest levels of stress. Administrative staff and ancillary workers such as food services and cleaning workers are not always visible as front-line workers, but also have patient-facing roles and therefore may be impacted by the pandemic situation. We postulate that research into HCW distress which will be used to support intervention strategies should encompass a broad range of staff categories.

Here, we present our protocol for a study, staff perceptions of COVID-19 healthcare system response by cancer care services, aiming to understand and document in real-time the perceptions of operational adjustments and resultant psychosocial impacts on a broad spectrum of staff from two large cancer care services in Southeast Queensland, Australia.

\section{METHODS AND ANALYSIS Setting}

This study is being conducted across two public teaching hospital networks in South east Queensland, Australia. Metro North Hospital and Health Service is a metropolitan, publicly funded healthcare provider network including a quaternary hospital (Royal Brisbane and Women's Hospital, RBWH) and several outreach centres in hospital and clinic settings. Sunshine Coast Hospital and Health Service (SCHHS) includes a tertiary referral centre (Sunshine Coast University Hospital, SCUH) and regional and rural outreach hospitals (Nambour General Hospital and Gympie Hospital, respectively).

RBWH is the largest referral hospital in the state, with over 900 inpatient beds, and a cancer care service which provides inpatient and outpatient services in medical oncology, radiation oncology, haematology and bone marrow transplant, and palliative care. SCUH is a regional tertiary hospital situated approximately $100 \mathrm{~km}$ north of RBWH. SCUH has over 550 inpatient beds and a cancer care service which provides medical oncology, radiation oncology, haematology and palliative care at SCUH and several regional hospitals. Queensland is the Northeastern state of Australia, which has a relatively large proportion of its population living outside major capital cities including in remote areas and very low population density statewide.

Although tailored to the institutions currently involved in the study, the protocol is flexible and as such has the 
potential to be used in other hospitals both state-wide and nationally as the pandemic evolves.

\section{Study design}

This study has two complimentary arms: (1) prospective real-time documentation of organisational changes communicated to staff during COVID-19 and (2) a concurrent HCW survey, distributed electronically at intervals, exploring the psychosocial impacts due to these changes.

\section{Diarising organisational changes}

Two principal investigators are independently documenting operational changes relating to adaptations of the cancer care and health service due to COVID-19 as they are relayed to staff in real time. These investigators will keep an individual diary of events pertaining to the pandemic response. Operational changes are recorded under the following executional domains: prospective diary of events, clinical trials unit, inpatient oncology/ haematology ward developments and outpatient developments. The strategy of prospectively documenting events was derived from the Toronto experience. ${ }^{17}$

Prospective diarising of events includes email communications from various sources such as government and hospital executives and recording structural changes (such as establishment of fever clinics and changes to outpatient services). Daily adjustments will include any departmental, hospital, state or national directive or communication pertinent to either the pandemic response or to the care of cancer patients. Examples would include implementation of lockdown measures, mandatory facemask use, limitation to visitors/nonessential staff or treatment stratification. The inclusion of directives to the diary will be deliberately broad in order to capture the range of changes that occur.

\section{HCW survey study participants}

Concurrently, a prospective online survey of HCW is being conducted. Included participants of the study are HCW currently employed by our health services and engaged in the setting of cancer care (self-defined as those whose main day-to-day activities take place in cancer care) who provide written informed consent. Staff who are unlikely to continue in that role for the greater duration of the pandemic (defined pragmatically as at least 6 months) were excluded. In order to include a spectrum of staff involved in cancer care, we included five groups based on the general tasks conducted by those staff: Administration, Allied Health and Other Health Professionals (eg, medical physicists), Ancillary Staff and Other (eg, food service workers, cleaning services), Nursing, and Medical. A minimum of ten participants per group at RBWH and five per group at SCUH was sought, to ensure participant confidentiality; this sample size was chosen in a pragmatic fashion and without a power calculation for a specific endpoint. No maximum number of participants was set. Additionally, staff were stratified according to site (RBWH or SCHHS), and their self-identified involvement in inpatient and/or outpatient, and/or community settings. Participants were initially approached via generic email to all staff mailing list, which was followed by face-toface interaction by study investigators in order to record consent. Individual written participant consent was sought via a participant information sheet and consent form, which was collected using the principles of Good Clinical Practice, including an option for future withdrawal of consent by any participant for any reason. Measures to minimise self-selection bias in the context of voluntary participation were the maintenance of confidentiality (ie, each survey being completed anonymously, the distribution email list being blinded, and the aggregation of results by category), and flexibility (ie, non-compulsory and non-tracked participation in each weekly survey).

\section{Survey development and pilot}

This prospective self-reported survey tool is hosted online by the public consultation tool which sits on a platform with the local ehealth network Citizen Space (Delib, Team Rubber, Bristol, UK) (online supplemental appendix 1).

Survey questions were agreed by consensus after study investigators, consisting of medical and nursing staff, consulted local HCW, informed by literature review of HCW experiences from a previous viral epidemic. ${ }^{17}{ }^{18}$ It underwent iterations of review before piloting. A preliminary questionnaire was piloted on 11 employees, from each of the staff work groups (administration, $n=3$; allied health and other health professionals, $\mathrm{n}=2$; ancillary staff, $\mathrm{n}=2$; nursing, $\mathrm{n}=2$; medical, $\mathrm{n}=2$ ) to check clarity, brevity and relevance. The final survey comprises four domains exploring staff well-being, departmental situation perception, self-reported experiences and reflections, reported using a Likert scale, with two open free text questions at the end. The Staff Well-Being section explores the themes of personal support, patient support and attending to HCW personal needs. The next section, Departmental Situation Perception asks participants to rate their perception on how well prepared the cancer care department was for the weeks' developments in relation to COVID-19. Following this, Your Experience This Past Week seeks to quantify the burden of COVID-19-related queries that the participant has dealt with in the week and their perception of their capability to respond to these. The study participants are asked to estimate the number of such queries which have originated from three sources: patients and their carers/families, staff and personal family member/members of the community. Queries can arise through any source, such as through telephone, email, in person or via social media. The participant is then invited to rate how equipped they felt to answer these queries. Finally, the last survey section, Reflections, provides an opportunity for the participant to share open free text responses about any reflections they have had in relation to COVID-19 (first, with respect to any difficult decision they have had to make, and second, any other reflections) and a self-reported distress tool adapted from 
the National Comprehensive Cancer Network distress thermometer. ${ }^{29}$ The free-text reflections will undergo interim analyses at 4-weekly timepoints throughout the study for content analysis and themes.

Individual responses are anonymised and nonidentifiable. The duration of the survey is dependent on the pandemic situation, with surveys distributed electronically on a weekly basis initially during the emergency response to COVID-19. However, given the dynamic nature of the pandemic, provision has been made for review of the protocol when required to assess extension, or curtailing of the study. Surveys are opened and distributed electronically each Friday, with 5 days given for responses. The ongoing schedule of the survey is likely to be variable, in order to prevent survey fatigue and will follow the Queensland Government pandemic plan. Participants will be kept informed of the schedule, as well as their obligations in the weekly email. A maximum end date for the study was set at 1 year following the commencement but could be truncated if COVID-19 is controlled into a recovery phase (characterised by low/ no community transmission and release of lockdown measures). Resources provided through the health service for employee support have been listed on the survey in the event of psychosocial distress arising from survey questions. This includes access to a 24/7 psychology/ counselling telephone service that is provided by the institution. Participants will be followed up with reminders of the availability of this service at intervals after completion of the survey, while also being updated on pertinent outcomes of the study (eg, dissemination of results).

\section{Data analysis}

Qualitative content analysis will be used to identify key themes for the reflective survey items in the staff survey. Content framework analysis will be done in relation to the above time periods to determine different themes which emerge over time. ${ }^{30}$ Investigators will independently code the data separately, develop, apply and pilot an analytical framework. Methodological rigour will be ensured through (1) memos, (2) dataset review, (3) memberchecking, (4) multiple and cross-coding. Using the final framework matrix data synthesis will be completed in iterations of review with extended research team. Due to the potentially highly sensitive nature of responses, a decision was taken to adopt methodology that looked at participants as broad groups rather than individuals in order to preserve anonymity. Because of this, participants will be unable to be tracked throughout the weekly surveys (no participant identifier available). Given that it will be unknown which participants have repeated measures, the analysis will be descriptive in nature, as inferential statistics would require methods that account for the correlation of observations within individuals. For semiquantitative survey items, medians and SD will be presented, comparing site of survey (RBWH vs SCHHS), type of employee (work group categories) and time period of pandemic response (preparation, execution and recovery). Interim analyses will be conducted to evaluate the data collection methods. Survey data collected will be presented in a topographical fashion and linked with the diarised events as they occurred. An attempt to temporally link events with the levels of distress, qualitative themes and quantitative variables will be made.

\section{Patient and public involvement}

No patients will be involved in this study.

\section{Ethics and dissemination}

Multisite ethical approval for the study was granted through the Human Research Ethics Committee (HREC) at RBWH (LNR/2020/QRBW/62982). Written consent for survey participants was mandated and was obtained by investigators prior to any study procedures, for example, completion of the survey. Information was included in the participant information form and each survey directing participants to counselling from our employee assistance provider in the event of distress caused by the survey subject matter.

This study, to our knowledge, represents the first attempt to document the effect of a healthcare crisis across a complete spectrum of staff in cancer care settings. The aim of the study is to provide knowledge and experience that assist in preparation of our institution and others for future pandemic events and provide insight into operational changes occurring across the entire time frame of the crisis thereby contributing to knowledge around recovery efforts. The circumstances surrounding the formulation of our present study required nimble conceptualisation of the study requirements and aims, due to the increasing momentum of the outbreak. Given the rapid spread of SARS-CoV-2, agile development of our protocol to enable timely commencement of the study was necessary.

On review, few publications addressing the wider staff involved in cancer care and very little data documenting the operational changes in the cancer care settings of previous viral epidemics/pandemics could be found. Current pandemic planning has also relied heavily on expert medical opinion. Several aspects of pandemic planning and recovery would benefit from prospective evidence such as the distress levels and burdens on all areas of the workforce, in order to support all workers through the crisis and during recovery. In particular, the cancer care workforce faces a unique set of stressors, including caring for a vulnerable population who often have unique supportive relationships with staff over a significant period and secondary effects of the pandemic (eg, restrictions in care provision) raising concerns for adverse future oncological outcomes. This combined with the distress from patients and their families/carers, and from members of one's own family/community, is likely to cause significant psychosocial distress on some members of the cancer care workforce that should be prospectively documented.

Some steps in our planned protocol have required careful negotiation. Given the potentially distressing nature 
of events that are predicted to unfold, it was agreed that highlighting the means of access to a counselling helpline via our employee assistance provider should be provided as part of the survey. Additionally, we found that pilottesting the survey was useful, as this assisted with clarifying questions, resolved some ambiguities and suggested some qualitative themes for further contemplation. As our survey was designed to encompass staff from multiple workforce groups, endorsement from several departments and groups (nursing, pharmacy, allied health) in the cancer care workforce was required prior to the commencement of recruitment to the study.

Selection of participants has been a key consideration in designing this study, as our unique angle has been to encompass members of the cancer care team from roles outside of medical practitioners. Consequently, we have asked our participants to indicate their main area of work. Initially our plan had been to assign groups according to individual role, for example, cleaning services. However, confidentiality of responses cannot be guaranteed with this approach and thus to mitigate this, we elected to aggregate the cohort into five groups. The five areas that we have chosen were decided through our focus group to cover distinct areas of practice but encompass the breadth of employees engaged in oncology services.

Participant recruitment has been an important consideration for our group, due to the potential for selection bias if the recruitment process was not transparent. Through utilising an open 'all-staff' email with expression of interest strategy, we aimed to eliminate the possibility of this. Our study has a minimum recruitment target, and to date over 150 participants have consented to take part, but beyond this we have not placed a cap on the number of participants; it will therefore be important to ensure that the different groups are well represented, and periodic review of survey responses will aid in this. Our study has an ambitious target for survey response, with an aim for weekly completion of the questionnaire over a 6-month period. This time course is deliberately long, in order to provide an opportunity to capture data from each phase of the pandemic; preparation, response, transition and recovery. Response fatigue is a potential risk and may increase as the situation progresses. Careful use of reminder emails should help to ameliorate this, and in addition, we have provisioned for review of the responses as the circumstances evolve allowing us to be dynamic and adjust the study accordingly.

COVID-19 has provided an existential challenge, and a realisation of the potentially precipitous nature of our way of life. As our species continues to interact on a global scale, threats such as the rapid spread of new pathogens are inevitable. Continuing to add to the knowledge of pandemic responses is therefore vital and enriches our approach to future events. As our systems improve their capacity to limit the mortality of a pandemic event, the psychosocial impact of threats such as this increases in importance. Our study hopes to provide fresh insights and a unique, inclusive methodology which incorporates staff from multiple backgrounds, reflecting our multidisciplinary approach to healthcare and cancer care.

Author affiliations

${ }^{1}$ Cancer Care Services, Royal Brisbane and Women's Hospital, Herston, Queensland, Australia

${ }^{2}$ School of Medicine, The University of Queensland, Saint Lucia, Queensland, Australia

${ }^{3}$ Surgery and Cancer Care, Darling Downs Hospital and Healthcare Service,

Toowoomba, Queensland, Australia

${ }^{4}$ Monash Health, Clayton, Victoria, Australia

${ }^{5}$ School of Clinical Sciences, Faculty of Medicine, Monash University, Clayton, Victoria, Australia

${ }^{6}$ School of Public Health and Social Work, Queensland University of Technology, Brisbane, Queensland, Australia

${ }^{7}$ Adem Crosby Cancer Centre, Sunshine Coast University Hospital, Sunshine Coast, Queensland, Australia

Acknowledgements The investigators would like to thank the Metro North Hospital and Health Service, and the Sunshine Coast Hospital and Health Services Cancer Care Services for their kind support in developing and approving this project, and in particular the HCW who have participated in the survey. The investigators would also like to acknowledge and thank the Safety and Quality Officers from RBWH Cancer Care for their support in publishing the weekly survey.

Contributors $\mathrm{HG}$ comprised the draft and final approved manuscript, contributed substantially to the conception, acquisition and design of the protocol. EA revised the draft for important intellectual content, and contributed substantially to the conception, acquisition and design of the protocol and approved the final draft. NR contributed significant revisions to the draft for important intellectual content, and contributed substantially to the conception, acquisition and design of the protocol as well as approval the final draft. $\mathrm{BC}$ contributed significant revisions to the draft for important intellectual content, and contributed substantially to the conception, acquisition and design of the protocol as well as approval the final draft. BH reviewed the initial draft for important intellectual content, provided substantial contributions to the design of the protocol and approved the final draft. GK reviewed the initial draft for important intellectual content, provided substantial contributions to the design of the protocol and approved the final draft. DW reviewed the initial draft for important intellectual content, provided substantial contributions to the design of the protocol and approved the final draft. ME reviewed the initial draft for important intellectual content, provided substantial contributions to the design of the protocol and approved the final draft. ZL is leader of the project and contributed significant revisions to the draft for important intellectual content, contributed substantially to the conception, acquisition and design of the protocol and gave approval of the final draft.

Funding The authors have not declared a specific grant for this research from any funding agency in the public, commercial or not-for-profit sectors.

Competing interests None declared.

Patient consent for publication Not required.

Provenance and peer review Not commissioned; externally peer reviewed.

Supplemental material This content has been supplied by the author(s). It has not been vetted by BMJ Publishing Group Limited (BMJ) and may not have been peer-reviewed. Any opinions or recommendations discussed are solely those of the author(s) and are not endorsed by BMJ. BMJ disclaims all liability and responsibility arising from any reliance placed on the content. Where the content includes any translated material, BMJ does not warrant the accuracy and reliability of the translations (including but not limited to local regulations, clinical guidelines, terminology, drug names and drug dosages), and is not responsible for any error and/or omissions arising from translation and adaptation or otherwise.

Open access This is an open access article distributed in accordance with the Creative Commons Attribution Non Commercial (CC BY-NC 4.0) license, which permits others to distribute, remix, adapt, build upon this work non-commercially, and license their derivative works on different terms, provided the original work is properly cited, appropriate credit is given, any changes made indicated, and the use is non-commercial. See: http://creativecommons.org/licenses/by-nc/4.0/.

\section{ORCID iD}

Harry Gasper http://orcid.org/0000-0003-0290-5762 


\section{REFERENCES}

1 Zhu N, Zhang D, Wang W, et al. A novel coronavirus from patients with pneumonia in China, 2019. N Engl J Med 2020;382:727-33.

2 Coronaviridae Study Group of the International Committee on Taxonomy of Viruses. The species severe acute respiratory syndrome-related coronavirus: classifying 2019-nCoV and naming it SARS-CoV-2. Nat Microbiol 2020;5:536-44.

3 Zhou F, Yu T, Du R, et al. Clinical course and risk factors for mortality of adult inpatients with COVID-19 in Wuhan, China: a retrospective cohort study. Lancet 2020;395:1054-62.

4 Liang W, Guan W, Chen R, et al. Cancer patients in SARSCoV-2 infection: a nationwide analysis in China. Lancet Oncol 2020;21:335-7.

5 Kim Y-J, Lee ES, Lee Y-S. High mortality from viral pneumonia in patients with cancer. Infect Dis 2019;51:502-9.

6 Schreiber RD, Old LJ, Smyth MJ. Cancer immunoediting: integrating immunity's roles in cancer suppression and promotion. Science 2011;331:1565-70.

7 Jazieh A-R, Alenazi TH, Alhejazi A, et al. Outcome of oncology patients infected with coronavirus. JCO Glob Oncol 2020;6:471-5.

8 Shalapour S, Karin M, Immunity KM. Immunity, inflammation, and cancer: an eternal fight between good and evil. J Clin Invest 2015;125:3347-55.

9 Onder G, Rezza G, Brusaferro S. Case-Fatality rate and characteristics of patients dying in relation to COVID-19 in Italy. JAMA 2020;323:1775-6.

10 Smith AC, Thomas E, Snoswell CL, et al. Telehealth for global emergencies: implications for coronavirus disease 2019 (COVID-19). $J$ Telemed Telecare 2020;26:309-13.

11 Australian Government Department of Health. Australian health sector emergency response plan for novel coronavirus (COVID-19), 2020. Available: https://www.health.gov.au/sites/default/files/ documents/2020/02/australian-health-sector-emergency-responseplan-for-novel-coronavirus-covid-19_2.pdf

12 Leong IY-O, Lee AO-K, Ng TW, et al. The challenge of providing holistic care in a viral epidemic: opportunities for palliative care. Palliat Med 2004;18:12-18.

13 FDA. Guidance on conduct of clinical trials of medical products during COVID-19 pandemic. guidance for industry, Investigators, and institutional review boards, 2020. Available: https://www.fda.gov/ media/136238/download

14 Sud A, Jones ME, Broggio J, et al. Collateral damage: the impact on outcomes from cancer surgery of the COVID-19 pandemic. Ann Oncol 2020;31:1065-74.

15 Gasper H, Ahern E, Roberts N, et al. COVID-19 and the cancer care workforce: from doctors to ancillary staff. Semin Oncol 2020;47:309-11.
16 Cortiula F, Pettke A, Bartoletti M, et al. Managing COVID-19 in the oncology clinic and avoiding the distraction effect. Ann Oncol 2020;31:553-5

17 Maunder R, Hunter J, Vincent L, et al. The immediate psychological and occupational impact of the 2003 SARS outbreak in a teaching hospital. CMAJ 2003;168:1245-51.

18 Tam CWC, Pang EPF, Lam LCW, et al. Severe acute respiratory syndrome (SARS) in Hong Kong in 2003: stress and psychological impact among frontline healthcare workers. Psychol Med 2004;34:1197-204.

19 Chan GCT, Koh D. Reviewing lessons learnt of SARS in Singapore during planning for influenza pandemic. Int Marit Health 2006;57:163-76.

20 Tay J, Ng YF, Cutter JL, et al. Influenza A (H1N1-2009) pandemic in Singapore--public health control measures implemented and lessons learnt. Ann Acad Med Singap 2010;39:313-12.

21 Greenberg N, Docherty M, Gnanapragasam S, et al. Managing mental health challenges faced by healthcare workers during covid-19 pandemic. BMJ 2020;368:m1211.

22 Jazieh A-R, Al Hadab A, Al Olayan A, et al. Managing oncology services during a major coronavirus outbreak: lessons from the Saudi Arabia experience. JCO Glob Oncol 2020;6:518-24.

23 Shankar A, Saini D, Roy S, et al. Cancer care delivery challenges amidst coronavirus disease - 19 (COVID-19) outbreak: specific precautions for cancer patients and cancer care providers to prevent spread. Asian Pac J Cancer Prev 2020;21:569-73.

24 Hanna TP, Evans GA, Booth CM, Cancer BCM. Cancer, COVID-19 and the precautionary principle: prioritizing treatment during a global pandemic. Nat Rev Clin Oncol 2020;17:268-70.

25 De Guzman R, Malik M. Dual challenge of cancer and COVID-19: impact on health care and socioeconomic systems in Asia Pacific. JCO Glob Oncol 2020;6:906-12.

26 Rizo CA, Lupea D, Baybourdy H, et al. What Internet services would patients like from hospitals during an epidemic? lessons from the SARS outbreak in Toronto. J Med Internet Res 2005;7:e46.

27 Skowronski DM, Petric M, Daly P, et al. Coordinated response to SARS, Vancouver, Canada. Emerg Infect Dis 2006;12:155-8.

28 Stewart J, Savage P, Johnson JC, et al. Telling the story of SARS: compassionate oncology care in the face of a futuristic health crisis. Can Oncol Nurs J 2005;15:175-83.

29 Hoffman BM, Zevon MA, D'Arrigo MC, et al. Screening for distress in cancer patients: the NCCN rapid-screening measure. Psychooncology 2004;13:792-9.

30 Gale NK, Heath G, Cameron E, et al. Using the framework method for the analysis of qualitative data in multi-disciplinary health research. BMC Med Res Methodol 2013;13:117. 